Article

\title{
Nutrient Intakes and Food Sources of Filipino Infants, Toddlers and Young Children are Inadequate: Findings from the National Nutrition Survey 2013
}

\author{
Liya Denney ${ }^{1, *}$, Imelda Angeles-Agdeppa ${ }^{2}$, Mario V. Capanzana ${ }^{2}$, Marvin B. Toledo ${ }^{2}$, \\ Juliana Donohue ${ }^{1}$ and Alicia Carriquiry ${ }^{3}$ \\ 1 Nestlé Research, Vers-chez-les-Blanc, Lausanne 1000, Switzerland; Juliana.Donohue@rd.nestle.com \\ 2 Food and Nutrition Research Institute, Department of Science and Technology, \\ Taguig City 1631, Philippines; iangelesagdeppa@yahoo.com.ph (I.A.-A.); \\ mar_v_c@yahoo.com (M.V.C.); marvinbtoledo@gmail.com (M.B.T.) \\ 3 Iowa State University, Ames, IA 50011, USA; alicia@iastate.edu \\ * Correspondence: liya.denney@rdls.nestle.com; Tel.: +41-21-785-8954
}

Received: 18 October 2018; Accepted: 9 November 2018; Published: 11 November 2018

\begin{abstract}
Comprehensive assessment of nutrient intakes and food sources of nutrients in Filipino children under 5 years old are lacking. We studied energy and nutrient intakes and food sources in 4218 children aged 6-59.9 months using two 24-h dietary recalls. Usual energy and nutrient intakes were estimated using the PC-SIDE program. Reported foods and beverages were assigned to one of 85 food groups. Percentage contribution of each food group to nutrient intake was calculated. The results showed that the intake of total fat as a percentage of energy and of most micronutrients were highly inadequate. The prevalence of inadequate nutrient intakes, defined as the percent of children with intakes less than the estimated average requirements (EAR) ranged from $60-90 \%$ for iron, calcium, vitamin $\mathrm{C}$, and zinc and ranged from $30-50 \%$ for others such as vitamin A, thiamine, riboflavin, niacin, vitamin $\mathrm{B} 6$, and phosphorus. The diets of these children were composed of limited foods, namely a large amount of refined rice and other low-nutrient-dense foods (cookies and sugar), while vegetables, fruits, meats, and eggs made little contribution to daily energy or nutrients. These findings provide direction to health professionals developing food-based recommendations and strategies to tackle the shortfalls in the diet of this population.
\end{abstract}

Keywords: usual nutrient intakes; food sources; infants; young children; The Philippines

\section{Introduction}

Nutrition in early life is crucially important for children to grow and develop into healthy adults. Children can reach their growth and development potential if their nutritional needs are met in a healthy environment [1-3]. Unfortunately, the Philippines is one of the countries in the world where a significant number of children remain malnourished despite the economic growth and development in the country over the past decades [4]. Data from the 2013 National Nutrition Survey (2013 NNS) in the Philippines reported that among children under 5 years old, the prevalence of malnutrition measured by underweight and stunting was $20 \%$ and $30 \%$, respectively, and the prevalence starts to increase at 6-11 months [5]. In addition, the prevalence of anemia among these children was $13.8 \%$. Infants aged 6-11 months had the highest prevalence of anemia at $40.5 \%$, and the prevalence of vitamin A deficiency was $27.9 \%$ [6].

Compared with other population groups, less is known about the dietary status of Filipino infants and toddlers, especially how subgroups of different ages vary in their nutrient intakes and food consumption during this critical growth period. There have been a number of studies focusing on 
certain nutrition topics and geographic areas in the Philippines. For example, macronutrient and selected vitamin intakes from complementary foods were investigated in infants and toddlers in the Cebu province. That study found that with the exception of protein, intakes of energy and vitamins for all age groups were below the World Health Organization estimated needs and desired nutrient densities from complementary foods [7]. Rohner and colleagues reported infant and young child feeding practices and their associations with stunting, anemia and deficiencies of iron and vitamin A in urban Philippines [8]. Other studies included determinants of anemia among preschool children from rural villages in Cebu and the use of dietary diversity scores as an indicator of micronutrient intakes in children aged 24-71 months [9-11].

Up to now, there has been no comprehensive assessment of nutrient intakes and food sources of nutrients in a nationally representative sample of infants and young children in the Philippines and surrounding countries in Southeast Asia, where the prevalence of malnutrition is also high [12]. Such a study is needed to demonstrate the proportion of the population that are not meeting dietary recommendations, as well as to provide insight on the relative contribution and importance of specific foods and food groups consumed in the population. This knowledge can provide a basis for population-level estimates and assist health professionals to form targeted measures to improve shortfalls. The purpose of this study was to use data from the NNS 2013 to describe the dietary intakes of infants, toddlers and young children aged from 6 months to 5 years including (1) the prevalence of inadequate nutrient intakes, (2) food groups consumed and (3) contribution of food groups to energy and nutrients.

\section{Methods}

\subsection{Study Population}

Data from 4218 infants, toddlers and young children aged 6-59.9 months who participated in the 2013 NNS were used in the current analyses. The 2013 NNS is a cross-sectional, population-based survey that characterizes the health and nutritional status of the Filipino population. The survey used a stratified three-stage sampling system drawn to represent all 17 regions and 80 provinces of the country covering both urban and rural areas. A total of 35,825 Filipino households were sampled with a response rate of $91 \%$. The Ethics Committee of Food Nutrition Research Institute (FNRI) approved the survey protocol and data collection instruments. All surveyed households provided informed consent prior to participation.

\subsection{Data Collection}

Two 24-h dietary recalls were conducted face-to-face with the parent or caregiver of each child. Trained registered dietitians carried out the interviews during household visits using a structured questionnaire. To estimate the day-to-day variance component in energy and nutrient intake required for usual intake analysis, the first 24-h dietary recall was collected for all children and a second 24-h dietary recall was repeated in $50 \%$ of randomly selected households on a non-consecutive day. The second 24-h dietary recall was typically collected 2 days after the first $24-\mathrm{h}$ recall. The interviewer asked about all foods and beverages that the child consumed on the previous day. The amount of each food item or beverage was estimated using common household measurements such as cups, tablespoons, by size or number of pieces. The information was then converted to grams using a portion to weight list for common foods compiled by FNRI or through actual weighing of the food samples.

Data on family economic status, weight and height of the children were taken from the same NNS 2013 reports. Briefly, family economic status was assessed by wealth quintiles defined as poorest, poor, middle, rich, and richest. The wealth status was defined by household possession of vehicles, gadgets and appliances [13]. The World Health Organization-Child Growth Standards were used to assess the nutritional status of children $0-60$ months based on weight and height measurements $[5,14]$. 


\subsection{Data Processing}

Food records were entered and estimated energy and nutrient intakes were processed with a computer system called Individual Dietary Evaluation System. This system contains the data of an updated Filipino Food Composition Tables (FCT) created for this study. The updated FCT contains 27 nutrients from 1359 foods. About half of the data (47\%) were from the United Sates Department of Agriculture (USDA) national nutrient database and 39\% of the data from the original Filipino FCT compiled by FNRI [15]. The rest of the data were sourced from the food composition database of Association of Southeast Asian Nations and other Asian countries such as Japan (8\%) and information from food labels (6\%). All imported data were adapted according to the International Network of Food Data Systems guidelines Food Agriculture Organization [16].

Quality control of the dietary intake data was conducted in two steps. In the first step, the foods reported by a participant were reviewed and information including coding and quantity reported. In the second step, energy and nutrient intakes were reviewed to identify implausible values by using the method described by Lopez-Olmedo and colleagues [17]. The estimated energy requirement (EER) was calculated for each individual by using the equations for maintenance of body weight from the Institute of Medicine based on age, gender, weight, height, and physical activity information [18]. We assumed a sedentary physical activity level for this study population. For implausible energy intake, the ratio of daily energy intake to EER was calculated for each person and transformed to a logarithmic scale to remove outliers below -3 SDs and above +3 SDs. For implausible micronutrient intakes, excessive intakes were defined as those that exceeded 1.5 times the $99^{\text {th }}$ percentile of the observed intake distribution of the nutrient in the corresponding sex and age group. Intakes above the upper limit were substituted by a random value generated from a uniform distribution in the intervals with the lower bound equal to the $95^{\text {th }}$ percentile of the observed intake and an upper bound equal to 1.5 times the $99^{\text {th }}$ percentile [17]. After data editing and processing, 47 individuals were excluded from the analysis for energy intake.

Breast milk consumption was estimated based on the child's age in months and total amount of other milks consumed (such as infant formula and cow's milk) using the information from published literature $[19,20]$. For infants aged 6 to 11.9 months fed human milk as the sole milk source, the amount of human milk was assumed to be $600 \mathrm{~mL} /$ day; for partially breastfed infants, the amount of human milk was computed as $600 \mathrm{~mL} /$ day minus the amount of infant formula/other milks consumed. For breastfed toddlers aged 12-23 months, the amount of human milk was computed as $529 \mathrm{~mL} /$ day minus the amount of infant formula/other milks consumed [21]. For toddlers aged 24-35.9 months, the amount of human milk was estimated as $59 \mathrm{~mL}$ per feeding occasion.

To investigate food groups consumed and food sources of energy and nutrients, a list of 85 food groups was created in a similar format from previous dietary intake studies in young children in the USA [22,23] (Table 1). Two trained Filipino nutritionists and a nutrition scientist at Nestlé Research adjusted food groups to incorporate local food culture and habits. Data analyses for food groups consumed and sources of energy and nutrients were based on the first 24-h dietary recall. All foods and beverages reported were assigned to one of the 85 food groups. Fortified milk powder produced for toddlers and young children in the Philippines is called toddler and preschooler formula in this study. 
Table 1. Food group classification.

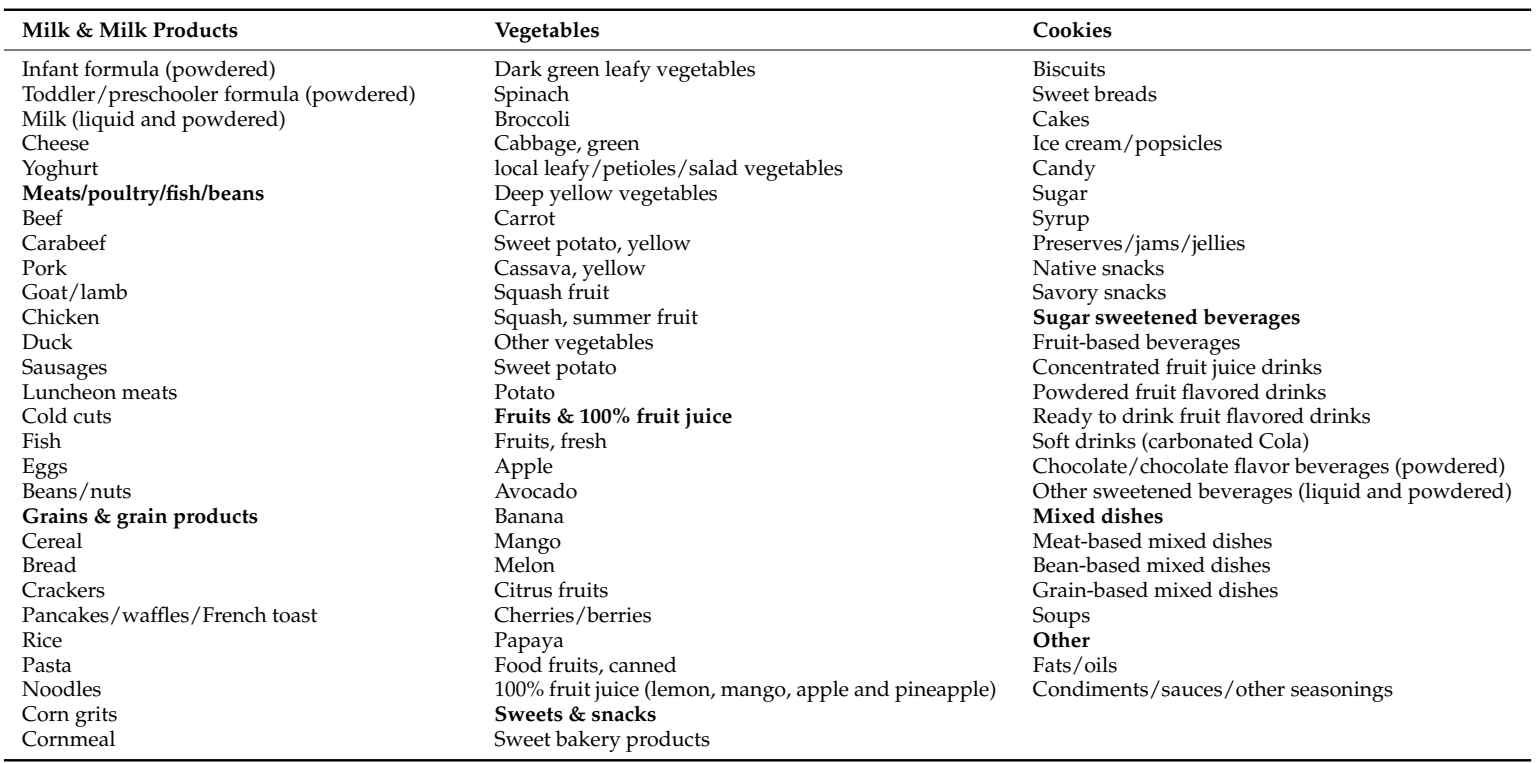

\subsection{Statistical Analysis}

Mean and usual intake distributions of energy and nutrients were estimated using the software developed by Iowa State University, PC-SIDE version 1.02 (Iowa State University, Ames, IA, USA) and within-person variation of nutrient intake was accounted for across days. This program estimates distributions (in percentiles) of usual nutrient intake by removing the effect of day-to-day (intra-person) variability in intake from daily intakes, and then calculating the proportion below the estimated average requirements (EAR) defined by the Philippine Dietary Reference Intakes 2015 [24]. Hence, the prevalence of inadequacy in the population is estimated as the proportion of individuals with usual intakes below the EAR [25].

Acceptable Macronutrient Distribution Ranges (AMDR) were used to evaluate carbohydrates, total fat, and protein intakes as a percentage of energy. The proportions of inadequate and excessive intakes were classified as less than the AMDR lower range and greater than AMDR upper range, respectively. For the prevalence of inadequate intakes of iron, a probability approach was used [26]. First, the risk of inadequacy of each individual was computed and then the prevalence of inadequate iron intake was calculated, which is the average risk of inadequacy. Calculations for summary statistics were carried out using STATA version 13 (StataCorp, College Station, TX, USA).

Food group consumption was expressed as the percentage of children who consumed specific food groups at least once on the first 24-h dietary recall regardless of the amount consumed. This method has been used in our previous studies [22,27]. The weighted percentage contribution of each food group for selected key nutrients was calculated by adding the amount of a given nutrient provided by each food group for all individuals and dividing by the total intake of that nutrient consumed by all individuals from all foods and beverages. In order to understand food consumption in detail and how it changes with age, data for nutrient intakes, food groups and sources of nutrients are presented for four age groups: 6-11 months, 12-23.9 months, 24-35.9 months and 36-59.9 months.

\section{Results}

\subsection{Demographic Charateristics of the Study Population}

The characteristics of children, mothers and family wealth status are shown in Table 2. Approximately $50 \%$ of the participants were boys and $50 \%$ were girls. Fifty-six percent of the children were from urban regions and the rest were from rural regions. Half of the families $(50.7 \%)$ were classified as poor and poorest. Among the mothers, nearly $77 \%$ had an education above high school 
and $71.2 \%$ did not have an occupation outside the home. The majority of the mothers were married or lived with their partner (89.7\%). The prevalence of underweight and stunting among the children included in this study were $21.1 \%$ and $29.3 \%$ respectively while prevalence of wasting and overweight were $5.7 \%$ and $3.9 \%$ respectively.

Table 2. Characteristics of the study population.

\begin{tabular}{|c|c|c|c|}
\hline & & $n$ & $\%$ \\
\hline \multirow[t]{2}{*}{ Gender } & Boy & 2143 & 50.3 \\
\hline & Girl & 2121 & 49.7 \\
\hline \multirow[t]{4}{*}{ Age } & 6-11.9 month & 362 & 8.5 \\
\hline & 12-23.9 month & 734 & 17.2 \\
\hline & 24-35.9 month & 741 & 17.4 \\
\hline & 36-59.9 months & 2427 & 56.9 \\
\hline \multirow[t]{5}{*}{ Wealth quintiles } & Poorest & 1224 & 29.5 \\
\hline & Poor & 880 & 21.2 \\
\hline & Middle & 820 & 19.8 \\
\hline & Rich & 684 & 16.5 \\
\hline & Richest & 542 & 13.1 \\
\hline \multirow[t]{2}{*}{ Region } & Urban & 2378 & 55.8 \\
\hline & Rural & 1886 & 44.2 \\
\hline \multirow[t]{5}{*}{ Underweight } & 6-11.9 month & 54 & 1.3 \\
\hline & 12-23.9 month & 128 & 3.0 \\
\hline & 24-35.9 month & 160 & 3.8 \\
\hline & 36-59.9 months & 543 & 12.9 \\
\hline & All & 885 & 21.1 \\
\hline \multirow[t]{5}{*}{ Stunted } & 6-11.9 month & 52 & 1.2 \\
\hline & 12-23.9 month & 198 & 4.7 \\
\hline & $24-35.9$ month & 235 & 5.6 \\
\hline & 36-59.9 months & 740 & 17.7 \\
\hline & All & 1225 & 29.3 \\
\hline \multirow[t]{5}{*}{ Wasted } & 6-11.9 month & 43 & 1.0 \\
\hline & 12-23.9 month & 50 & 1.2 \\
\hline & 24-35.9 month & 40 & 1.0 \\
\hline & 36-59.9 months & 105 & 2.5 \\
\hline & All & 239 & 5.7 \\
\hline \multirow[t]{5}{*}{ Overweight } & 6-11.9 month & 14 & 0.4 \\
\hline & 12-23.9 month & 29 & 0.9 \\
\hline & 24-35.9 month & 29 & 0.9 \\
\hline & 36-59.9 months & 62 & 1.8 \\
\hline & All & 134 & 3.9 \\
\hline \multirow[t]{5}{*}{ Mother's marital status } & Single & 227 & 6.7 \\
\hline & Married & 2325 & 68.7 \\
\hline & Live-in & 712 & 21.1 \\
\hline & Separated/divorced/widowed & 117 & 3.4 \\
\hline & Unknown & 2 & 0.1 \\
\hline \multirow[t]{6}{*}{ Mother's education } & No grade completed & 52 & 1.2 \\
\hline & Elementary level & 828 & 21.7 \\
\hline & High school level & 1655 & 49.1 \\
\hline & Vocational level & 158 & 4.9 \\
\hline & College level & 794 & 22.9 \\
\hline & Others & 7 & 0.02 \\
\hline \multirow[t]{3}{*}{ Mother's current work } & No occupation & 2426 & 71.2 \\
\hline & Student & 24 & 0.01 \\
\hline & With job/business & 920 & 28.1 \\
\hline
\end{tabular}

\subsection{Nutrient Intakes and Food Sources of Infants (6-11.9 Month Olds)}

The mean energy intake of $688 \mathrm{kcal} /$ day was $7.8 \%$ higher than the estimated EER (mean $\pm \mathrm{SE}$ ): $638 \pm 6 \mathrm{kcal} /$ day. Fifteen percent of the infants had percentage of energy from total fat below while 
$36 \%$ exceeded the AMDR in this age group (Table 3). High prevalence of inadequate intakes were found for vitamin A $(84 \%)$, iron (76\%) and zinc $(63 \%)$. Considerable high prevalence of inadequate intakes were found for protein $(43 \%)$, thiamine $(52 \%)$, riboflavin $(45 \%)$ and niacin $(58 \%)$. Mean intakes of vitamin C, vitamin B6, folate and magnesium were above the AIs while mean intakes of vitamin E, phosphorus, vitamin D and potassium were far below the AIs. (Table 2). Mean sodium intake $(303 \mathrm{mg} / \mathrm{d})$ was above the AI $(200 \mathrm{mg} / \mathrm{d})$.

Consumption of the top-20 most consumed food groups and their contribution to energy and selected nutrient intakes are presented in Table 4. Refined rice, human milk, infant formula, cookies and cow's milk were the top-5 foods most consumed followed by infant cereal, vegetables, grain-based mixed dishes, table sugar and fish (Table 4). Fruits were only consumed by $5.5 \%$ of the infants.

Human milk, infant formula, rice, cow's milk and infant cereal were the top-5 sources of energy and most of the selected key nutrients (Table 4). Besides the top-5 foods, the proportion of infants consuming other food groups was low and their contribution to nutrient intakes was small except for toddler/preschooler formula. Although toddler/preschooler formula was only consumed by $3.7 \%$ of the infants, it was among the top four or five sources of total fat, vitamin A, vitamin C, calcium and iron, indicating many other food groups contributed very little to nutrient intakes (Table 4).

\subsection{Nutrient Intakes and Food Sources of Younger Toddlers (12-23.9 Month Olds)}

Mean energy intake of $771 \mathrm{kcal} /$ day just met the estimated EER (mean $\pm \mathrm{SE}): 784 \pm 6 \mathrm{kcal} /$ day. High prevalence of inadequate intakes were found for iron (78\%), folate $(68 \%)$, vitamin B6 (61\%), vitamin A (60\%), and calcium (62\%). Inadequacy was also found for thiamine (59\%), riboflavin $(42 \%)$, niacin (57\%), vitamin B12 (53), phosphorus (57\%), and zinc (52\%) (Table 5). Mean intakes of vitamin E, vitamin D and potassium were all far below the AIs but mean sodium intake $(517 \mathrm{mg} / \mathrm{d})$ was about twofold higher than the AI $(225 \mathrm{mg} / \mathrm{d})$.

Refined rice, human milk, cow's milk, fish, and vegetables were the top- 5 foods most consumed, although vegetables were only consumed by $28 \%$ of the children (Table 6 ). The next five food groups were cookies, toddler/preschooler formula, bread, noodles, and eggs.

Among the younger toddlers, refined rice, human milk, cow's milk, toddler/preschooler formula, and cookies were among the top- 5 sources of energy. The above first four foods together with sugar-sweetened beverages were the top-5 sources of selected key nutrients (Table 6). Briefly, refined rice was the first source of protein and the third or fourth source of thiamine, riboflavin, iron, zinc and calcium. Cow's milk was the first source of riboflavin, vitamin A and calcium. Toddler/preschooler formula was the first source of thiamine, vitamin C, iron and zinc. Sugar-sweetened beverages were the third to fifth sources of thiamine, riboflavin, vitamin A, calcium, iron and zinc (Table 6). Like infants, other food groups were consumed by small percentages of the children and contributed little to nutrient intakes overall. 
Table 3. Usual energy and nutrient intakes from food and beverages for Filipino infants aged 6-11.9 months $(n=350)$.

\begin{tabular}{|c|c|c|c|c|c|c|c|c|c|c|c|}
\hline \multirow[b]{2}{*}{ Nutrients } & \multicolumn{3}{|c|}{ Dietary Reference Intakes ${ }^{1}$} & \multicolumn{5}{|c|}{ Mean/Median Intake Percentiles } & \multicolumn{3}{|c|}{ Inadequate/Excessive Reported Intake } \\
\hline & EAR/AMDR & AI/RNI & UL & 10th & 25th & Median & Mean \pm SE & 75th & 90th & $\%<$ EAR/AMDR & $\%>$ AMDR/>UL \\
\hline Macronutrients & & & & & & & & & & & \\
\hline Energy intake (kcal/day) & 638 (EER) & & & 443 & 497 & 610 & $688 \pm 14$ & 815 & 1066 & - & - \\
\hline Total fat $(\mathrm{g} / \mathrm{d})$ & - & - & - & 18.9 & 21.6 & 24.7 & $24.5 \pm 0.5$ & 30.7 & 40.8 & - & - \\
\hline Protein $(\mathrm{g} / \mathrm{d})$ & 13.5 & - & - & 14.8 & 11 & 14.8 & $18.6 \pm 0.6$ & 23 & 32.9 & 43 & - \\
\hline Carbohydrate (g/d) & - & - & - & 53 & 63 & 80 & $90.6 \pm 2$ & 109 & 145 & - & - \\
\hline Total sugars $(\mathrm{g} / \mathrm{d})$ & - & - & - & 6.1 & 26.1 & 31.7 & $34.9 \pm 1.2$ & 42.4 & 60.8 & - & - \\
\hline Dietary fiber $(\mathrm{g} / \mathrm{d})$ & - & - & - & 0.1 & 0.3 & 0.7 & $1.3 \pm 0.1$ & 1.6 & 2.7 & - & - \\
\hline \multicolumn{12}{|l|}{ As percentage of total energy } \\
\hline Total Fat $(\%)$ & $30-40$ & - & - & 27.5 & 33.2 & 37.9 & $36.7 \pm 0.4$ & 41.5 & 44.1 & 15 & 36 \\
\hline Carbohydrate (\%) & $45-62$ & - & - & 45.3 & 48.6 & 51.7 & $52.9 \pm 0.4$ & 55.9 & 61.4 & 9 & 9 \\
\hline Antioxidants & & & & & & & & & & & \\
\hline Vitamin C (mg/d) & - & - & & 21.9 & 26.2 & 34.8 & $54.6 \pm 2.3$ & 72 & 120.5 & - & - \\
\hline $\begin{array}{l}\text { Vitamin } E(\mathrm{mg} / \mathrm{d}) \\
\text { B vitamins }\end{array}$ & - & 4 & - & 0.3 & 0.5 & 1.1 & $2.8 \pm 0.2$ & 3.6 & 8.4 & - & - \\
\hline Thiamine (mg/d) & 0.3 & - & - & 0.1 & 0.1 & 0.3 & $0.5 \pm 0.03$ & 0.7 & 1.2 & 52 & - \\
\hline Riboflavin (mg/d) & 0.3 & - & - & 0.1 & 0.1 & 0.4 & $0.8 \pm 0.1$ & 1.3 & 2 & 45 & - \\
\hline Niacin (mg/d) & 3.5 & - & - & 1 & 1.4 & 2.8 & $3.9 \pm 0.2$ & 5.5 & 8.4 & 58 & - \\
\hline Vitamin B6 (mg/d) & - & 0.25 & - & 0.1 & 0.1 & 0.2 & $0.3 \pm 0.03$ & 0.5 & 1 & - & - \\
\hline Folate (DFE $\mu \mathrm{g} / \mathrm{d})$ & - & 75 & - & 26 & 37 & 63 & $95.6 \pm 5$ & 122 & 217 & - & - \\
\hline Vitamin B12 ( $(\mathrm{gg} / \mathrm{d})$ & - & - & - & 0.2 & 0.4 & 0.6 & $1.1 \pm 0.1$ & 1.4 & 2.7 & - & - \\
\hline \multicolumn{12}{|l|}{ Bone-related nutrients } \\
\hline Magnesium (mg/d) & - & 50 & - & 17 & 24 & 37 & $53.1 \pm 2.6$ & 65 & 112 & - & - \\
\hline Vitamin $D(\mu \mathrm{g} / \mathrm{d})$ & - & 5 & 25 & 0.5 & 0.9 & 1.6 & $4.3 \pm 0.3$ & 5.4 & 12 & - & - \\
\hline \multicolumn{12}{|l|}{ Other micronutrients } \\
\hline Vitamin A ( $\mu \mathrm{g}$ RE/d) & 190 & - & 600 & 3.8 & 25.6 & 51.3 & $126 \pm 12$ & 126.3 & 269 & 84 & \\
\hline $\operatorname{Iron}(\mathrm{mg} / \mathrm{d})$ & 8.4 & - & & 1.6 & 2.2 & 3.7 & $6.5 \pm 0.4$ & 8.1 & 15.5 & 76 & - \\
\hline Zinc $(\mathrm{mg} / \mathrm{d})$ & 2.65 & - & 5 & 0.9 & 1.3 & 1.9 & $3 \pm 0.1$ & 3.8 & 7 & 63 & 18 \\
\hline Sodium $(\mathrm{mg} / \mathrm{d})$ & - & 200 & 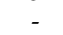 & 126 & 167 & 245 & $303 \pm 10$ & 379 & 567 & - & - \\
\hline Potassium (mg/d) & - & 700 & & 245 & 338 & 435 & $579 \pm 22$ & 694 & 1149 & - & - \\
\hline Selenium $(\mu \mathrm{g} / \mathrm{d})$ & 7.75 & - & 60 & 6.6 & 12.2 & 15.7 & $18.6 \pm 0.7$ & 22.4 & 33.5 & 12 & 1 \\
\hline
\end{tabular}

${ }^{1}$ Philippine Dietary Reference Intakes 2015. EAR, estimated average requirements. AMDR, acceptable macro-nutriment distribution range. AI, adequate intake. RNI, reference nutrient intake. UL, tolerable upper intake level. DFE, dietary folate equivalents. RE, retinol equivalents. 
Table 4. Top-20 most consumed food groups and their contribution to energy and selected nutrient intakes among infants aged 6-11 months ( $n=350)$ in the Philippines.

\begin{tabular}{|c|c|c|c|c|c|c|c|c|c|c|c|c|c|c|}
\hline \multirow[b]{3}{*}{ Rank } & \multirow[b]{3}{*}{ Food Groups } & \multirow[b]{3}{*}{$\%$ of Children } & \multirow[b]{3}{*}{ Mean Intake (SE) Per Capita (g) } & \multicolumn{11}{|c|}{ Percent Contribution to Total Daily Intake (Ranking of Top 5 Food Sources of Nutrients) } \\
\hline & & & & \multicolumn{4}{|c|}{ Macronutrients } & \multicolumn{4}{|c|}{ Vitamins } & \multicolumn{3}{|c|}{ Minerals } \\
\hline & & & & Energy & Carbohydrate & Protein & Total Fat & Thiamine & Riboflavin & Vitamin A & Vitamin C & Calcium & Iron & Zinc \\
\hline 1 & Rice & 70.0 & $35.2(7.6)$ & $11.7(3)$ & $19.9(3)$ & $8.9(4)$ & 0.5 & $5.9(5)$ & $1.8(5)$ & 0 & 0 & 1.3 & $3.7(4)$ & $6.6(3)$ \\
\hline 2 & Human milk & 60.0 & $336.1(5.4)$ & 33.9 (1) & $28.5(1)$ & $25.8(2)$ & $43.9(1)$ & $7.6(3)$ & $4.5(4)$ & $6.7(3)$ & $26.3(2)$ & $17.3(3)$ & $16.9(3)$ & $21.2(2)$ \\
\hline 3 & Infant formula & 35.1 & $38.6(3.7)$ & $27.6(2)$ & $24.8(2)$ & 30.5 (1) & $31.5(2)$ & $55.0(1)$ & $52.9(1)$ & $60.6(1)$ & $56.3(1)$ & $50.2(1)$ & 44.5 (1) & 44.1 (1) \\
\hline 4 & Cookies & 25.4 & $3.6(0.5)$ & 2.4 & 3.0 & 1.4 & 1.9 & 0.1 & 0.7 & 0.3 & 0 & 0.5 & 0.7 & 0.8 \\
\hline 5 & Cow's milk & 14.9 & $12.2(4.5)^{1}$ & $8.2(4)$ & $4.7(5)$ & $15.5(3)$ & $11.2(3)$ & $6.5(4)$ & $25.2(2)$ & $22.9(2)$ & $2.9(4)$ & $18.9(2)$ & 0.6 & $6.5(4)$ \\
\hline 6 & Infant cereal & 14.3 & $7.6(2.6)$ & $4.3(5)$ & $5.0(4)$ & $5.2(5)$ & $2.5(4)$ & $12.6(2)$ & $7.5(3)$ & 0.6 & $8.7(3)$ & $5.5(4)$ & $24.0(2)$ & $6.2(5)$ \\
\hline 7 & Vegetables & 11.4 & $2.3(1.1)$ & 0.3 & 0.4 & 0.2 & 0 & 0.5 & 0.2 & 1.0 & 0.7 & 0.2 & 0.3 & 0.2 \\
\hline 8 & Grain-based mixed dishes & 10.3 & $12.5(7.5)$ & 1.4 & 2.0 & 1.0 & 0.5 & 0.3 & 0.4 & 0 & 0 & 0.2 & 0 & 1.7 \\
\hline 9 & Table sugar & 10.0 & $1.6(1.2)$ & 0.9 & 1.7 & 0 & 0 & 0 & 0.1 & 0 & 0 & 0.4 & 0.1 & 0 \\
\hline 10 & Fish & 9.7 & $1.5(0.9)$ & 0.2 & 0 & 1.5 & 0.2 & 0.2 & 0.3 & 0.9 & 0 & 0.4 & 0.4 & 0.6 \\
\hline 11 & Eggs & 9.7 & $1.3(0.7)$ & 0.3 & 0 & 0.9 & 0.5 & 0.2 & 0.7 & 0.9 & 0 & 0.1 & 0.4 & 0.7 \\
\hline 12 & Crackers & 8.6 & $1.1(0.4)$ & 0.8 & 0.8 & 0.5 & 0.9 & 0.4 & 0.2 & 0 & 0 & 0.2 & 0.3 & 0.3 \\
\hline 13 & Bread & 8.3 & $2.0(1.1)$ & 1.0 & 1.4 & 1.2 & 0.3 & 1.2 & 0.4 & 0.2 & 0 & 0.2 & 1.1 & 0.6 \\
\hline 14 & Sugar sweetened beverages & 6.6 & $2.8(2.8)^{2}$ & 0.5 & 0.8 & 0.2 & 0.1 & 1.9 & 1.0 & $1.3(5)$ & 1.3 & 0.4 & 0.9 & 0.9 \\
\hline 15 & Fruits & 5.4 & $3.1(2.5)$ & 0.5 & 0.9 & 0.2 & 0 & 0.3 & 0.1 & 0.3 & 1.5 & 0.1 & 0.3 & 0.2 \\
\hline $\begin{array}{l}16 \\
17\end{array}$ & Cakes & 5.1 & $1.0(0.6)$ & 0.6 & 0.7 & 0.3 & 0.5 & 0.4 & 0.1 & 0.4 & 0 & 0.2 & 0.4 & 0.3 \\
\hline${ }_{18}^{17}$ & $\begin{array}{l}\text { Noodles } \\
\text { Whitecong }\end{array}$ & 5.1 & $0.7(0.7)$ & 0.5 & 0.5 & 0.4 & 0.4 & 0.7 & 0.1 & 0 & 0 & 0 & 0.2 & 0.3 \\
\hline $\begin{array}{l}18 \\
19\end{array}$ & $\begin{array}{l}\text { White corn } \\
\text { Sausages/luncheon meats }\end{array}$ & $\begin{array}{l}4.3 \\
4.3\end{array}$ & $\begin{array}{l}1.4(1.3) \\
1 .(1.8)\end{array}$ & 0.7 & 1.2 & $\begin{array}{l}0.6 \\
10\end{array}$ & $\begin{array}{l}0.1 \\
0.9\end{array}$ & 0.2 & 0.1 & $\begin{array}{c}0 \\
03\end{array}$ & $\begin{array}{l}0 \\
0\end{array}$ & $\begin{array}{c}0 \\
01\end{array}$ & $\begin{array}{l}0.1 \\
13\end{array}$ & 0.4 \\
\hline \multirow{2}{*}{\multicolumn{2}{|c|}{$\begin{array}{l}{ }^{20} \text { Toddler/preschooler formula } \\
\text { Total contribution of top } 20 \text { foods }\end{array}$}} & 3.7 & $\begin{array}{l}1.4(1.0) \\
3.0(3.19\end{array}$ & 1.3 & 1.2 & 1.7 & $1.3(5)$ & 2.3 & 2.3 & $\begin{array}{l}0.3 \\
1.6(4)\end{array}$ & $2.1(5)$ & $2.9(5)$ & $2.2(5)$ & 5.1 \\
\hline & & & & 97.6 & 97.6 & 97.0 & 97.2 & 96.5 & 98.8 & 98.0 & 99.8 & 99.1 & 98.4 & 97.5 \\
\hline
\end{tabular}

${ }^{1}$ includes $10.7 \%$ liquid milk and $89.3 \%$ milk powder. ${ }^{2}$ includes $86 \%$ liquid beverages and $14 \%$ beverage powder. Grey shadow and bold words highlight the top-five food sources of nutrients. 
Table 5. Usual energy and nutrient intakes from food and beverages for Filipino toddlers aged 12-23.9 months $(n=714)$.

\begin{tabular}{|c|c|c|c|c|c|c|c|c|c|c|c|}
\hline \multirow[b]{2}{*}{ Nutrients } & \multicolumn{3}{|c|}{ Dietary Reference Intakes ${ }^{1}$} & \multicolumn{5}{|c|}{ Mean/Median Intake Percentiles } & \multicolumn{3}{|c|}{ Inadequate/Excessive Reported Intak } \\
\hline & EAR/AMDR & AI/RNI & UL & 10th & 25th & Median & Mean $\pm \mathrm{SE}$ & 75th & 90th & $\%<$ EAR/AMDR & $\%>$ AMDR/ $>$ UL \\
\hline \multicolumn{12}{|l|}{ Macronutrients } \\
\hline Energy intake (kcal/day) & 784 (EER) & & & 444 & 560 & 717 & $771 \pm 12$ & 918 & 1159 & - & - \\
\hline Total fat $(\mathrm{g} / \mathrm{d})$ & - & - & - & 11.3 & 17.5 & 23.8 & $25.6 \pm 0.5$ & 31.3 & 42 & - & - \\
\hline Saturated fat $(\mathrm{g} / \mathrm{d})$ & - & - & - & 2.3 & 4.4 & 7.9 & $8.5 \pm 0.2$ & 11 & 14.6 & - & - \\
\hline Protein (g/d) & 14.5 & - & - & 11.2 & 15 & 20.8 & $23.3 \pm 0.4$ & 28.8 & 38.6 & 23 & - \\
\hline Carbohydrate $(\mathrm{g} / \mathrm{d})$ & - & - & - & 63 & 80 & 102 & $112 \pm 2$ & 132 & 169 & - & - \\
\hline Total sugars (g/d) & - & - & - & 6 & 17 & 29 & $31.6 \pm 0.8$ & 41 & 59 & - & - \\
\hline Dietary fiber $(\mathrm{g} / \mathrm{d})$ & - & $6-7$ & - & 0.7 & 1.2 & 2 & $2.3 \pm 0.1$ & 3.1 & 4.5 & - & - \\
\hline \multicolumn{12}{|l|}{ As percentage of total energy } \\
\hline Total Fat $(\%)$ & $25-35^{\text {a }}$ & - & - & 18 & 24.1 & 30.3 & $29.6 \pm 0.3$ & 35.7 & 40.1 & 28 & 28 \\
\hline Protein $(\%)$ & $6-15^{a}$ & - & - & 8.6 & 9.8 & 11.5 & $11.9 \pm 0.1$ & 13.6 & 15.6 & $<1$ & 13 \\
\hline Carbohydrate (\%) & $50-69^{\text {a }}$ & - & - & 47.1 & 52 & 57.6 & $58.4 \pm 0.3$ & 64.3 & 70.9 & 18 & 13 \\
\hline Antioxidants & & & & & & & & & & & \\
\hline Vitamin $C(\mathrm{mg} / \mathrm{d})$ & 11.5 & - & 400 & 5.3 & 14.2 & 24.5 & $31.9 \pm 1.1$ & 37.7 & 69 & 21 & 0 \\
\hline \multirow{2}{*}{\multicolumn{12}{|c|}{$\begin{array}{l}\text { Vitamin } \mathrm{E}(\mathrm{mg} / \mathrm{d}) \\
\text { B vitamins }\end{array}$}} \\
\hline & & & & & & & & & & & \\
\hline Thiamine (mg/d) & 0.4 & - & - & 0.1 & 0.2 & 0.3 & $0.4 \pm 0.01$ & 0.6 & 0.9 & 59 & - \\
\hline Riboflavin (mg/d) & 0.4 & & - & 0.1 & 0.2 & 0.5 & $0.8 \pm 0.03$ & 1.1 & 1.9 & 42 & - \\
\hline Niacin (mg/d) & 5 & - & 10 & 1.8 & 2.8 & 4.5 & $5.3 \pm 0.1$ & 7 & 10 & 57 & 10 \\
\hline Vitamin B6 (mg/d) & 0.45 & - & 30 & 0.1 & 0.2 & 0.3 & $0.6 \pm 0.05$ & 0.6 & 1.2 & 61 & $<1$ \\
\hline Folate (DFE $\mu \mathrm{g} / \mathrm{d}$ ) & 120 & - & - & 28 & 47 & 81 & $223 \pm 3$ & 142 & 236 & 68 & - \\
\hline Vitamin B12 ( $\mu \mathrm{g} / \mathrm{d})$ & 0.85 & - & - & 0.3 & 0.4 & 0.8 & $1.1 \pm 0.03$ & 1.5 & 2.3 & 53 & - \\
\hline \multicolumn{12}{|l|}{ Bone-related nutrients } \\
\hline Calcium $(\mathrm{mg} / \mathrm{d})$ & 440 & - & 2500 & 121 & 179 & 321 & $479 \pm 16$ & 640 & 1058 & 62 & $<1$ \\
\hline Phosphorus (mg/d) & 380 & - & 3000 & 148 & 210 & 334 & $424 \pm 11$ & 551 & 826 & 57 & 0 \\
\hline Magnesium (mg/d) & - & 60 & 65 & 22 & 32 & 48 & $57.1 \pm 1.4$ & 72 & 105 & - & - \\
\hline Vitamin D $(\mu \mathrm{g} / \mathrm{d})$ & - & 5 & 50 & 0.1 & 0.5 & $\begin{array}{l}\text { to } \\
1.3\end{array}$ & $2.3 \pm 0.1$ & 3 & 6.2 & - & 0 \\
\hline \multicolumn{12}{|l|}{$\begin{array}{l}\text { Vitamin } D(\mu \mathrm{g} / \mathrm{d}) \\
\text { Other micronutrients }\end{array}$} \\
\hline Vitamin A ( $\mu \mathrm{g} \mathrm{RE} / \mathrm{d})$ & 186.5 & - & 600 & 27 & 64 & 138 & $231 \pm 10$ & 307 & 554 & 60 & 8 \\
\hline $\operatorname{Iron}(\mathrm{mg} / \mathrm{d})$ & 6.7 & - & - & 1.5 & 2.3 & 3.6 & $4.9 \pm 0.1$ & 6.2 & 10.1 & 78 & - \\
\hline Zinc $(\mathrm{mg} / \mathrm{d})$ & $\begin{array}{l}.7 \\
2.7\end{array}$ & - & 7 & 1.3 & 1.7 & $\begin{array}{l}3.0 \\
2.6\end{array}$ & $4.2 \pm 0.2$ & $\begin{array}{l}0.2 \\
4.5\end{array}$ & 8.1 & 52 & 13 \\
\hline Sodium (mg/d) & - & 225 & - & 189 & 288 & 438 & $517 \pm 13$ & 654 & 933 & - & - \\
\hline Potassium (mg/d) & - & 700 & & 231 & 322 & 453 & $539 \pm 13$ & 663 & 953 & - & - \\
\hline Selenium $(\mu \mathrm{g} / \mathrm{d})$ & 13.3 & 100 & 90 & 12.8 & 18.2 & 26.1 & $30.5 \pm 0.7$ & 38 & 53.5 & 11 & 1 \\
\hline
\end{tabular}

${ }^{1}$ Philippine Dietary Reference Intakes 2015. EAR, estimated average requirements. AMDR, acceptable macronutrient distribution range. AI, adequate intake. RNI, reference nutrient intake. UL, tolerable upper intake level. DFE, dietary folate equivalents. RE, retinol equivalents. 
Table 6. Top-20 most consumed food groups and their contribution to energy and selected nutrient intakes among toddlers aged $12-23$ months $(n=714)$ in the Philippines.

\begin{tabular}{|c|c|c|c|c|c|c|c|c|c|c|c|c|c|c|}
\hline \multirow[b]{3}{*}{ Rank } & \multirow[b]{3}{*}{ Food Groups } & \multirow[b]{3}{*}{$\%$ of Children } & \multirow[b]{3}{*}{ Mean Intake (SE) Per Capita [g] } & \multicolumn{11}{|c|}{ Percent Contribution to Total Daily Intake (Ranking of Top 5 Food Sources of Nutrients) } \\
\hline & & & & \multicolumn{4}{|c|}{ Macronutrients } & \multicolumn{4}{|c|}{ Vitamins } & \multicolumn{3}{|c|}{ Minerals } \\
\hline & & & & Energy & Carbohydrate & Protein & Total Fat & Thiamine & Riboflavin & Vitamin A & Vitamin C & Calcium & Iron & Zinc \\
\hline 1 & Rice & 91.2 & $62.3(4.3)$ & $24.3(1)$ & $36.1(1)$ & $16.1(1)$ & 1.1 & $12.2(3)$ & $3.6(4)$ & 0 & 0 & $3.0(4)$ & $10.9(3)$ & $12.2(3)$ \\
\hline 2 & Human milk & $\begin{array}{l}31.2 \\
37.0\end{array}$ & $\begin{array}{l}0.31 .7(3.4) \\
181)\end{array}$ & $15.9(2)$ & $12.1(2)$ & $10.9(2)$ & $25.1(1)$ & $4.2(5)$ & 2.3 & $3.5(5)$ & $23.1(2)$ & $\begin{array}{l}3.0(4) \\
9.7(3)\end{array}$ & $\begin{array}{l}0.9(3) \\
11.3(2)\end{array}$ & $\begin{array}{l}9.4(4) \\
9\end{array}$ \\
\hline 3 & Cow's milk & 35.2 & $\begin{array}{l}23.0(2.2)^{1} \\
2\end{array}$ & $12.7(3)$ & $6.8(4)$ & $10.9(3)$ & $20.9(2)$ & $\begin{array}{l}4.2(1) \\
13.0(2)\end{array}$ & $43.4(1)$ & $\begin{array}{l}.03010 \\
36.9(1)\end{array}$ & $8.4(3)$ & 35.7 (1) & 2.4 & $\begin{array}{l}.74(4) \\
11.0(2)\end{array}$ \\
\hline 4 & Fish & 34.7 & $8.4(0.6)$ & 1.2 & $\begin{array}{c}0.0 \text { (4) } \\
0\end{array}$ & $7.5(5)$ & 1.1 & 1.4 & 130.6 & 3.3 & $\begin{array}{c}0.4(1) \\
0\end{array}$ & 2.4 & 2.4 & 3.0 \\
\hline 5 & Vegetables & 27.6 & $6.4(0.6)$ & 0.5 & 0.7 & 0.6 & 0.1 & 1.4 & 0.9 & $4.9(4)$ & 4.1 & 0.8 & 1.3 & 0.4 \\
\hline 6 & Cookies & 24.8 & $4.9(0.5)$ & $2.9(5)$ & $3.1(5)$ & 1.4 & 2.9 & 1.3 & 0.9 & 0.6 & 0 & 0.7 & 1.5 & 0.8 \\
\hline 7 & Toddler/preschooler formula & 22.8 & $23.3(2.7)$ & 11.4 (4) & $10.1(3)$ & $12.8(4)$ & $13.4(3)$ & $22.8(1)$ & $22.9(2)$ & $20.1(2)$ & $30.3(1)$ & $30.0(2)$ & $29.5(1)$ & $33.0(1)$ \\
\hline 8 & Bread & 21.1 & $6.0(0.8)$ & 2.5 & 3.2 & 2.7 & 0.9 & 3.4 & 1.2 & 0.3 & 0 & 0.6 & 3.8 & 1.6 \\
\hline 9 & Noodles & 20.3 & $4.6(0.9)$ & 2.6 & 2.6 & 2.0 & 2.8 & 4.0 & 0.7 & 0.3 & 0.2 & 0.2 & 1.7 & 1.6 \\
\hline 10 & Eggs & 19.9 & $5.4(0.8)$ & 1.0 & 0.1 & 2.9 & 2.1 & 1.0 & $2.8(5)$ & 3.1 & 0 & 0.4 & 2.0 & 2.2 \\
\hline 11 & Sugar sweetened beverages & 19.6 & $14.3(2.7)^{2}$ & 2.1 & 3.0 & 0.9 & 0.7 & $12.0(4)$ & $4.1(3)$ & $7.1(3)$ & $7.4(4)$ & $2.7(5)$ & $5.4(4)$ & $4.9(5)$ \\
\hline 12 & Table sugar & 19.3 & $3.1(0.8)$ & 1.6 & 2.7 & 0 & 0 & 0 & 0.2 & 0 & 0 & 0.8 & 0.2 & 0 \\
\hline 13 & Crackers & 18.1 & $3.9(0.6)$ & 2.4 & 2.2 & 1.5 & $3.2(5)$ & 1.9 & 0.9 & 0 & 0 & 0.5 & 1.4 & 0.9 \\
\hline 14 & Fruits & 14.4 & $9.2(2.4)$ & 1.0 & 1.6 & 0.4 & 0.2 & 0.8 & 0.5 & 0.8 & $7.4(5)$ & 0.8 & 1.2 & 0.4 \\
\hline 15 & Sausages/luncheon meats & 12.7 & $5.6(1.4)$ & 2.0 & 0.3 & 3.6 & $4.6(4)$ & 1.2 & 1.1 & 1.2 & 0 & 0.4 & $5.1(5)$ & 3.2 \\
\hline 16 & Pork & 9.7 & $2.7(0.7)$ & 1.0 & 0.1 & 1.8 & 2.6 & 2.4 & 0.8 & 2.9 & 0.1 & 0.2 & 0.9 & 2.6 \\
\hline 17 & Chicken & 9.7 & $2.4(0.7)$ & 0.5 & 0 & 2.0 & 0.8 & 0.3 & 0.3 & 0.3 & 0.1 & 0.3 & 0.6 & 0.9 \\
\hline 18 & Cakes & 9.4 & $3.6(1.1)$ & 1.7 & 1.8 & 0.9 & 1.9 & 1.5 & 0.5 & 1.4 & 0 & 0.8 & 1.7 & 0.8 \\
\hline 19 & $\begin{array}{l}\text { Grain-based mixed dishes } \\
\text { without meat }\end{array}$ & 9.1 & $17.3(5.8)$ & 1.8 & 2.3 & 1.1 & 0.7 & 0.4 & 0.6 & 1.2 & 0 & 0.3 & 0 & 1.9 \\
\hline \multirow{2}{*}{\multicolumn{2}{|c|}{$\begin{array}{l}20 \\
\text { White corn } \\
\text { Total contribution of top } 20 \text { foods }\end{array}$}} & 6.6 & $3.9(1.6)$ & 1.8 & 2.7 & 1.4 & 0.2 & 0.7 & 0.3 & 0 & 0 & 0.1 & 0.3 & 0.8 \\
\hline & & & & 90.9 & 91.5 & 81.4 & 85.3 & 85.9 & 89.6 & 87.9 & 81.1 & 90.4 & 83.6 & 91.6 \\
\hline
\end{tabular}

${ }^{1}$ includes $13.9 \%$ liquid milk and $86.1 \%$ milk powder. ${ }^{2}$ includes $82.5 \%$ liquid beverages and $17.5 \%$ beverage powder. Grey shadow and bold words highlight the top-five food sources of nutrients. 


\subsection{Nutrient Intakes and Food Sources of Older Toddlers (24-35.9 Month Olds)}

Mean energy intake, $838 \mathrm{kcal} /$ day, was about $13 \%$ below the estimated EER (mean $\pm \mathrm{SE}$ ): $962 \pm 7 \mathrm{kcal} /$ day. A high proportion, $58 \%$ of the older toddlers, consumed a low percentage of energy from total fat (Table 7). Similar to younger toddlers but with slightly lower proportions, a high prevalence of inadequate intakes were found for almost all of the vitamins and minerals: iron $(75 \%)$, calcium $(66 \%)$, folate $(63 \%)$, thiamine $(46 \%)$, phosphorus $(44 \%)$, zinc $(46 \%)$, vitamin A $(41 \%)$, vitamin B6 (40\%), vitamin C (35\%), riboflavin (35\%), niacin (30\%) and vitamin B12 (29\%). Mean intakes of vitamin E, vitamin D and potassium were all far below the AIs while the mean sodium intake $(646 \mathrm{mg} / \mathrm{d})$ was more than twofold higher than the AI $(225 \mathrm{mg} / \mathrm{d})$ (Table 7).

Among the older toddlers, refined rice, fish, vegetables, cow's milk and sugar-sweetened beverages were the top-five foods most consumed followed by table sugar, bread, noodles, eggs and cookies (Table 8 ). Fruits were consumed by $17 \%$ and toddler/preschooler formula by $15 \%$ of the children.

Refined rice, cow's milk, toddler/preschooler formula, bread, and noodles were top-5 sources of energy. For nutrients, refined rice, cow's milk, sugar-sweetened beverages and toddler/preschooler formula and pork were the top-5 sources (Table 8). Notably, sugar-sweetened beverages were consumed more in this age group (33\%) and became top sources of all the selected nutrients (Table 8 ). Other food groups such as vegetables, bread, noodles and fruits were among top- 5 sources for some nutrients. For example, vegetables were the fifth sources of vitamins $A$ and $C$ and fruits were the third source of vitamin C (Table 8).

\subsection{Nutrient Intakes and Food Sources of Young Children (36-59.9 Month Olds)}

Mean energy intake, $997 \mathrm{kcal} /$ day, was $11 \%$ below the estimated EER (mean \pm SE): $1119 \pm 2 \mathrm{kcal} /$ day. Twenty-seven percent of the children consumed a low proportion of energy from total fat (Table 9). High prevalence of inadequate intakes were found for iron (90\%), calcium $(84 \%)$, vitamin C (60\%), folate (72\%), zinc (47\%), thiamine (43\%) riboflavin (43\%) and vitamin A (43\%). Mean intakes of vitamin E, vitamin D and potassium were all far below the AIs while mean sodium intake $(740 \mathrm{mg} / \mathrm{d})$ was more than twofold higher than the AI (300 mg/d) (Table 9).

In this age group, refined rice, fish, vegetables, sugar-sweetened beverages, and cow's milk were the top- 5 foods most consumed followed by table sugar, bread, eggs, noodles, and pork (Table 10). For the sources of energy and nutrients, refined rice, cow's milk, bread, sugar-sweetened beverages, and noodles were the top- 5 sources of energy with rice alone, contributing $41 \%$. Rice was also the first source of protein, thiamine, iron and zinc. Sugar-sweetened beverages were the first source of vitamin $\mathrm{C}$ and the second source of thiamine, riboflavin, vitamin A, and iron. Cow's milk was the first to fifth source of all nutrients except iron (Table 10). Other foods among the top sources of nutrients were bread, pork, chicken, and fruits. Although fruits were only consumed by $17 \%$ of the children, they were the second highest source of vitamin C. 
Table 7. Usual energy and nutrient intakes from food and beverages for Filipino toddlers aged 24-35.9 months $(n=727)$.

\begin{tabular}{|c|c|c|c|c|c|c|c|c|c|c|c|}
\hline \multirow[b]{2}{*}{ Nutrients } & \multicolumn{3}{|c|}{ Dietary Reference Intakes $^{1}$} & \multicolumn{5}{|c|}{ Mean/Median Intake Percentiles } & \multicolumn{3}{|c|}{ Inadequate/Excessive Reported Intake } \\
\hline & EAR/AMDR & AI/RNI & UL & 10th & 25th & Median & Mean \pm SE & 75th & 90th & $\%<$ EAR/AMDR & $\%>$ AMDR/>UL \\
\hline \multicolumn{12}{|l|}{ Macronutrients } \\
\hline Energy intake (kcal/day) & 962 (EER) & & & 454 & 591 & 783 & $839 \pm 13$ & 1025 & 1294 & - & - \\
\hline Total fat $(\mathrm{g} / \mathrm{d})$ & - & - & - & 8.1 & 12.8 & 20.3 & $23.3 \pm 0.5$ & 30.5 & 42.5 & - & - \\
\hline Saturated fat $(\mathrm{g} / \mathrm{d})$ & - & - & - & 3 & 4.5 & 6.9 & $8.8 \pm 0.3$ & 10.7 & 16.3 & - & - \\
\hline Protein $(\mathrm{g} / \mathrm{d})$ & 14.5 & - & - & 13.1 & 18 & 25 & $27.5 \pm 0.5$ & 34.3 & 44.8 & 14 & - \\
\hline Carbohydrate $(\mathrm{g} / \mathrm{d})$ & - & - & - & 73 & 93 & 122 & $129 \pm 2$ & 157 & 196 & - & - \\
\hline Total sugars (g/d) & - & - & - & 7 & 13 & 24 & $28.8 \pm 0.8$ & 39 & 57 & - & - \\
\hline Dietary fiber $(\mathrm{g} / \mathrm{d})$ & - & $6-7$ & - & 1.6 & 2.3 & 3.2 & $3.5 \pm 0.1$ & 4.4 & 6.5 & - & - \\
\hline \multicolumn{12}{|l|}{ As percentage of total energy } \\
\hline Total Fat $(\%)$ & $25-35^{\text {a }}$ & - & - & 12.7 & 17.4 & 23.1 & $23.4 \pm 0.3$ & 29.2 & 34.4 & 58 & 9 \\
\hline Protein $(\%)$ & $6-15^{a}$ & - & - & 9.7 & 11.1 & 12.7 & $12.9 \pm 0.1$ & 14.6 & 16.5 & $<1$ & 21 \\
\hline $\begin{array}{l}\text { Carbohydrate }(\%) \\
\text { Antioxidants }\end{array}$ & $50-69^{\mathrm{a}}$ & - & - & 50.8 & 57.1 & 63.9 & $63.6 \pm 0.4$ & 70.4 & 76 & 9 & 30 \\
\hline $\begin{array}{c}\text { Antioxidants } \\
\text { Vitamin } C(\mathrm{mg} / \mathrm{d})\end{array}$ & 11.5 & - & 400 & 4.5 & 9 & 16 & $23.1 \pm 0.8$ & 29.4 & 49.8 & 35 & 0 \\
\hline Vitamin E (mg/d) & - & - & - & 0.5 & 0.9 & 1.6 & $2 \pm 0.1$ & 2.7 & 4.1 & - & - \\
\hline Thiamine (mg/d) & 0.4 & - & - & 0.2 & 0.3 & 0.4 & $0.5 \pm 0.01$ & 0.6 & 0.9 & 46 & - \\
\hline Riboflavin (mg/d) & 0.4 & & - & 0.2 & 0.3 & 0.6 & $0.8 \pm 0.03$ & 1 & 1.7 & 35 & - \\
\hline Niacin (mg/d) & 5 & - & 10 & 3.3 & 4.6 & 6.5 & $7.1 \pm 0.1$ & 9 & 11.1 & 30 & 18 \\
\hline Vitamin B6 (mg/d) & 0.45 & - & 30 & 0.2 & 0.3 & 0.5 & $0.8 \pm 0.04$ & 0.9 & 1.6 & 40 & 0 \\
\hline Folate (DFE $\mu \mathrm{g} / \mathrm{d})$ & 120 & - & - & 36 & 59 & 96 & $115 \pm 3$ & 150 & 216 & 63 & - \\
\hline Vitamin B12 ( $\mu \mathrm{g} / \mathrm{d})$ & 0.85 & - & - & 0.5 & 0.8 & 1.3 & $1.6 \pm 0.04$ & 2 & 3 & 29 & - \\
\hline \multicolumn{12}{|l|}{ Bone-related nutrients } \\
\hline Calcium (mg/d) & 440 & - & 2500 & 82 & 148 & 295 & $421 \pm 14$ & 565 & 936 & 66 & $<1$ \\
\hline Phosphorus (mg/d) & 380 & - & 3000 & 191 & 278 & 414 & $476 \pm 10$ & 605 & 838 & 44 & 0 \\
\hline Magnesium (mg/d) & - & 60 & 65 & 33 & 46 & 64 & $71.5 \pm 1.4$ & 91 & 118 & - & - \\
\hline \multirow{2}{*}{\multicolumn{12}{|c|}{ Other micronutrients }} \\
\hline & & & & & & & & & & & \\
\hline Vitamin A ( $\mu \mathrm{g} \mathrm{RE} / \mathrm{d})$ & 186.5 & - & 600 & 54 & 115 & 229 & $365 \pm 18$ & 430 & 772 & 41 & 15 \\
\hline $\operatorname{Iron}(\mathrm{mg} / \mathrm{d})$ & 6.7 & - & - & 1.9 & 2.7 & 4.2 & $5.2 \pm 0.1$ & 6.6 & 9.8 & 75 & - \\
\hline Zinc (mg/d) & 2.7 & - & 7 & 1.3 & 1.9 & 2.9 & $5 \pm 0.1$ & 4.3 & 6.2 & 46 & 7 \\
\hline Sodium (mg/d) & - & 225 & - & 252 & 381 & 572 & $646 \pm 14$ & 827 & 1130 & - & - \\
\hline Potassium (mg/d) & - & 700 & & 256 & 352 & 497 & $555 \pm 11$ & 693 & 926 & - & - \\
\hline Selenium $(\mu \mathrm{g} / \mathrm{d})$ & 13.3 & - & 90 & 18 & 26 & 36 & $38.9 \pm 0.7$ & 59 & 64 & 3 & 2 \\
\hline
\end{tabular}

${ }^{1}$ Philippine Dietary Reference Intakes 2015. EAR, estimated average requirements. AMDR, accept intake. UL, tolerable upper intake level. DFE, dietary folate equivalents. RE, retinol equivalents. 
Table 8. Top-20 most consumed food groups and their contribution to energy and selected nutrient intakes among toddlers aged $24-35.9$ months $(n=727)$ in the Philippines.

\begin{tabular}{|c|c|c|c|c|c|c|c|c|c|c|c|c|c|c|}
\hline \multirow[b]{3}{*}{ Rank } & \multirow[b]{3}{*}{ Food Groups } & \multirow[b]{3}{*}{$\%$ of Children } & \multirow[b]{3}{*}{ Mean Intake Per Capita (g) } & \multicolumn{11}{|c|}{ Percent contribution to total daily intake (ranking of top 5 food sources of nutrients) } \\
\hline & & & & \multicolumn{4}{|c|}{ Macronutrients } & \multicolumn{4}{|c|}{ Vitamins } & \multicolumn{3}{|c|}{ Minerals } \\
\hline & & & & Energy & Carbohydrate & Protein & Total Fat & Thiamine & Riboflavin & Vitamin A & Vitamin C & Calcium & Iron & Zinc \\
\hline 1 & Rice & 96 & $76.8(2.1)$ & $33.1(1)$ & $47.6(1)$ & $20.9(1)$ & 1.8 & $15.3(1)$ & $5.0(4)$ & 0 & 0 & $5.2(4)$ & $15.7(1)$ & $18.5(1)$ \\
\hline 2 & Fish & 50 & $16.9(1.1)$ & 2.3 & 0.1 & $12.8(3)$ & 2.6 & 2.5 & 3.2 & 5.8 & 0 & $5.5(3)$ & 4.4 & 4.7 \\
\hline 3 & Vegetables & 39 & $13.9(1.2)$ & 1.2 & 1.6 & 1.0 & 0.3 & 2.4 & 1.5 & $8.3(5)$ & $11.7(5)$ & 1.9 & 3.0 & 1.1 \\
\hline 4 & Cow's milk & 38 & $25.4(2.3)^{1}$ & $12.6(2)$ & $6.4(2)$ & $19.4(2)$ & $25.0(1)$ & $13.0(3)$ & $45.9(1)$ & $34.8(1)$ & $11.8(4)$ & $43.4(1)$ & 2.3 & $11.7(3)$ \\
\hline 5 & Sugar sweetened beverages & 33 & $29.8(3.4)^{2}$ & 3.6 & 4.9 & 1.3 & 1.4 & $16.6(3)$ & $7.2(3)$ & $10.1(3)$ & $23.6(1)$ & $5.1(5)$ & $8.7(3)$ & $7.9(4)$ \\
\hline 6 & Table sugar & 29 & $3.3(0.5)$ & 1.6 & 2.5 & 0 & 0 & 0 & 0.2 & 0 & 0 & 0.9 & 0.2 & 0 \\
\hline 7 & Bread & 28 & $12.0(1.1)$ & $4.9(4)$ & $5.9(3)$ & 4.7 & 2.2 & 6.0 & 2.3 & 1.0 & 0 & 1.5 & $7.7(4)$ & 3.3 \\
\hline 8 & Noodles & 27 & $7.6(0.9)$ & $3.9(5)$ & $3.8(5)$ & 2.8 & $5.1(5)$ & $6.6(5)$ & 1.6 & 0.2 & 0.2 & 0.4 & 2.8 & 2.6 \\
\hline 9 & Eggs & 25 & $5.3(0.7)$ & 1.3 & 3.1 & 3.5 & 3.3 & 1.1 & $4.0(5)$ & 3.9 & 0 & 0.6 & 2.6 & 3.0 \\
\hline 10 & Cookies & 20 & $5.3(0.7)$ & 3.1 & 2.1 & 1.3 & 4.0 & 1.3 & 1.0 & 0.8 & 0 & 0.9 & 1.8 & 0.9 \\
\hline 11 & Fruits & 17 & $11.0(2.0)$ & 1.1 & 1.7 & 0.4 & 0.2 & 1.2 & 0.7 & 0.7 & $17.0(3)$ & 0.8 & 1.5 & 0.5 \\
\hline 12 & Pork & 16 & $7.4(1.4)$ & 2.7 & 0.1 & $\begin{array}{c}4.7 \\
5.65\end{array}$ & $8.2(3)$ & 5.7 & 2.9 & $12.1(2)$ & 0.6 & 0.4 & 2.8 & $7.2(5)$ \\
\hline 13 & Chicken & 16 & $7.4(1.4)$ & 1.3 & 0 & $5.6(5)$ & 2.5 & 0.8 & 1.7 & 3.4 & 0.6 & 1.1 & 2.0 & 3.3 \\
\hline 14 & Toddler/preschooler formula & 15 & $12.6(2.2)$ & $5.8(3)$ & $4.7(4)$ & $5.7(4)$ & $8.7(2)$ & $11.2(4)$ & $12.5(2)$ & $9.3(4)$ & $22.0(2)$ & $20.5(2)$ & $15.7(2)$ & $18.1(2)$ \\
\hline 15 & Sausages/luncheon meats & 14 & $7.3(1$ & 2.3 & 0.5 & 3.8 & $6.5(4)$ & 1.4 & 1.2 & 1.9 & 0 & 0.5 & $6.5(5)$ & 4.2 \\
\hline 16 & Crackers & 14 & $3.5(0.5)$ & 2.1 & 1.8 & 1.2 & 3.4 & 1.5 & 0.8 & 0 & 0 & 0.5 & 1.3 & 0.8 \\
\hline 17 & Human milk & 11 & $9.9(0.4)$ & 0.8 & 0.6 & 0.5 & 1.6 & 0.2 & 0.1 & 0.2 & 1.8 & 0.6 & 0.6 & 0.5 \\
\hline 18 & Cakes & 11 & $4.5(1.2)$ & 2.1 & 2.1 & 1.0 & 2.7 & 1.7 & 0.5 & 1.7 & 0 & 1.0 & 2.1 & 0.9 \\
\hline 19 & Candy & 10 & $2.2(0.5)$ & 1.3 & 1.3 & 0.5 & 1.6 & 0.3 & 0.4 & 0.2 & 0 & 1.0 & 0.7 & 1.0 \\
\hline \multirow{2}{*}{\multicolumn{2}{|c|}{$\begin{array}{l}\text { Beans, nuts \& peas } \\
\text { Total contribution of top } 20 \text { foods }\end{array}$}} & 6 & $1.6(1.4)$ & $\begin{array}{l}0.6 \\
877\end{array}$ & $\begin{array}{l}0.5 \\
91.3\end{array}$ & $\begin{array}{c}1.1 \\
92.2\end{array}$ & $\begin{array}{l}0.4 \\
815\end{array}$ & 1.3 & $\begin{array}{c}0.3 \\
93 .\end{array}$ & 0 & 0.3 & 0.7 & 1.0 & 0.6 \\
\hline & & & & 87.7 & 91.3 & 92.2 & 81.5 & 90.1 & 93.0 & 94.4 & 89.6 & 92.5 & 83.4 & 90.8 \\
\hline
\end{tabular}

${ }^{1}$ includes $22.8 \%$ liquid milk and $77.2 \%$ milk powder. ${ }^{2}$ includes $75.8 \%$ liquid beverages and $24.2 \%$ beverage powder. Grey shadow and bold words highlight the top-five food sources of nutrients. 
Table 9. Usual energy and nutrient intakes from food and beverages for Filipino young children aged 36-59.9 months $(n=2427)$.

\begin{tabular}{|c|c|c|c|c|c|c|c|c|c|c|c|}
\hline \multirow[b]{2}{*}{ Nutrients } & \multicolumn{3}{|c|}{ Dietary Reference Intakes $^{1}$} & \multicolumn{5}{|c|}{ Mean/Median Intake Percentiles } & \multicolumn{3}{|c|}{ Inadequate/Excessive Reported Intake } \\
\hline & EAR/AMDR & AI/RNI & UL & 10th & 25th & Median & Mean $\pm \mathrm{SE}$ & 75th & 90th & $\%<$ EAR/AMDR & $\%>$ AMDR/>UL \\
\hline \multicolumn{12}{|l|}{ Macronutrients } \\
\hline Energy intake (kcal/day) & 1119 (EER) & & & 596 & 747 & 949 & $997 \pm 7$ & 1195 & 1458 & - & - \\
\hline Total fat $(\mathrm{g} / \mathrm{d})$ & - & - & - & 8 & 13 & 21 & $24.1 \pm 0.3$ & 31 & 44 & - & - \\
\hline Saturated fat $(\mathrm{g} / \mathrm{d})$ & 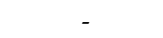 & - & - & 3 & 5 & 8 & $11 \pm 0.2$ & 13 & 21 & - & - \\
\hline Protein $(\mathrm{g} / \mathrm{d})$ & 17.5 & - & - & 18 & 23 & 30 & $31.5 \pm 0.3$ & 38 & 48 & 10 & - \\
\hline Carbohydrate $(\mathrm{g} / \mathrm{d})$ & - & - & - & 99 & 124 & 156 & $164 \pm 1$ & 195 & 237 & - & - \\
\hline Total sugars (g/d) & - & - & - & 8 & 15 & 25 & $28.8 \pm 0.4$ & 38 & 54 & - & - \\
\hline Dietary fiber $(\mathrm{g} / \mathrm{d})$ & - & $8-10$ & - & 2.7 & 3.4 & 4.5 & $4.9 \pm 0.04$ & 5.9 & 7.4 & - & - \\
\hline \multicolumn{12}{|l|}{ As percentage of total energy } \\
\hline Total Fat $(\%)$ & $15-30^{\mathrm{a}}$ & - & - & 10 & 15 & 20 & $20.2 \pm 0.2$ & 26 & 31 & 27 & 12 \\
\hline Protein $(\%)$ & $6-15^{a}$ & - & - & 10 & 11 & 13 & $12.7 \pm 0.04$ & 14 & 15 & 0 & 13 \\
\hline $\begin{array}{l}\text { Carbohydrate }(\%) \\
\text { Antioxidants }\end{array}$ & $55-79^{\mathrm{a}}$ & - & - & 55 & 61 & 68 & $67.1 \pm 0.2$ & 74 & 78 & 10 & 8 \\
\hline $\begin{array}{c}\text { Antioxidants } \\
\text { Vitamin } C(\mathrm{mg} / \mathrm{d})\end{array}$ & 17 & - & 650 & 5 & 8 & 14 & $16.9 \pm 0.3$ & 22 & 33 & 60 & 0 \\
\hline Vitamin E (mg/d) & & 5 & - & 0.9 & 1.4 & 2.1 & $2.4 \pm 0.03$ & 3 & 4.2 & - & - \\
\hline Thiamine (mg/d) & 0.45 & - & - & 0.25 & 0.35 & 0.49 & $0.54 \pm 0.01$ & 0.68 & 0.89 & 43 & - \\
\hline Riboflavin (mg/d) & 0.45 & - & - & 0.22 & 0.32 & 0.51 & $0.61 \pm 0.01$ & 0.78 & 1.12 & 43 & - \\
\hline Niacin (mg/d) & 5 & - & 15 & 4.8 & 6.3 & 8.4 & $8.92 \pm 0.07$ & 11 & 13.7 & 12 & 6 \\
\hline Vitamin B6 (mg/d) & 0.5 & - & 40 & 0.36 & 0.47 & 0.7 & $1.3 \pm 0.03$ & 1.36 & 2.85 & 29 & - \\
\hline Folate (DFE $\mu \mathrm{g} / \mathrm{d})$ & 160 & - & 400 & 55 & 80 & 119 & $123 \pm 3$ & 167 & 221 & 72 & 0 \\
\hline Vitamin B12 ( $\mu \mathrm{g} / \mathrm{d})$ & 0.95 & - & - & 1.03 & 1.39 & 1.94 & $2.1 \pm 0.02$ & 2.67 & 3.5 & 7 & - \\
\hline \multicolumn{12}{|l|}{ Bone-related nutrients } \\
\hline Calcium (mg/d) & 440 & - & 2500 & 107 & 156 & 239 & $286 \pm 4$ & 365 & 526 & 84 & 0 \\
\hline Phosphorus (mg/d) & 405 & - & 3000 & 262 & 346 & 461 & $493 \pm 4$ & 604 & 763 & 38 & 0 \\
\hline Magnesium (mg/d) & - & 70 & - & 51 & 66 & 85 & $91.1 \pm 0.7$ & 110 & 138 & - & - \\
\hline Vitamin $D(\mu \mathrm{g} / \mathrm{d})$ & - & 5 & - & 0.9 & 1.3 & 1.9 & $2.2 \pm 0.03$ & 2.8 & 3.9 & - & - \\
\hline \multicolumn{12}{|l|}{ Other micronutrients } \\
\hline Vitamin A ( $\mu \mathrm{g} \mathrm{RE/d)}$ & 220 & - & 900 & 104 & 161 & 244 & $279 \pm 3.5$ & 356 & 493 & 43 & 1 \\
\hline $\operatorname{Iron}(\mathrm{mg} / \mathrm{d})$ & 7.5 & - & - & 2.4 & 3.6 & 5 & $5.6 \pm 0.06$ & 6.9 & 9.3 & 90 & - \\
\hline Zinc (mg/d) & 3.25 & - & 12 & 1.9 & 2.5 & 3.4 & $4.8 \pm 0.1$ & 5.3 & 10.1 & 47 & 6 \\
\hline Sodium (mg/d) & - & 300 & - & 319 & 470 & 681 & $740 \pm 8$ & 946 & 1238 & - & - \\
\hline Potassium (mg/d) & - & 1400 & - & 366 & 465 & 607 & $647 \pm 5$ & 786 & 984 & - & - \\
\hline Selenium $(\mu \mathrm{g} / \mathrm{d})$ & 15.85 & - & 150 & 28 & 37 & 49 & $51.7 \pm 0.4$ & 63 & 79 & 1 & - \\
\hline
\end{tabular}

${ }^{1}$ Philippine Dietary Reference Intakes 2015. EAR, estimated average requirements. AMDR, accept intake. UL, tolerable upper intake level. DFE, dietary folate equivalents. RE, retinol equivalents. 
Table 10. Top-20 most consumed food groups and their contribution to energy and selected nutrient intakes among young children aged $36-59.9$ months $(n=2427)$ in the Philippines.

\begin{tabular}{|c|c|c|c|c|c|c|c|c|c|c|c|c|c|c|}
\hline \multirow[b]{3}{*}{ Rank } & \multirow[b]{3}{*}{ Food Groups } & \multirow[b]{3}{*}{$\%$ of Children } & \multirow[b]{3}{*}{ Mean Intake Per Capita (g) } & \multicolumn{11}{|c|}{ Percent Contribution to Total Daily Intake (Ranking of Top 5 Food Sources of Nutrients) } \\
\hline & & & & \multicolumn{4}{|c|}{ Macronutrients } & \multicolumn{4}{|c|}{ Vitamins } & \multicolumn{3}{|c|}{ Minerals } \\
\hline & & & & Energy & Carbohydrate & Protein & Total Fat & Thiamine & Riboflavin & Vitamin A & Vitamin C & Calcium & Iron & Zinc \\
\hline 1 & Rice & 93.2 & $114.5(0.6)$ & $41.2(1)$ & $55.5(1)$ & $27.0(1)$ & 2.8 & $21.3(1)$ & $8.8(3)$ & 0.0 & 0.0 & $10.8(3)$ & $21.6(1)$ & $26.0(1)$ \\
\hline 2 & Fish & 55.7 & $23.5(0.4)$ & 2.7 & 0.1 & $15.5(2)$ & 3.4 & 2.9 & $\begin{array}{l}5.3(5) \\
5.3(5)\end{array}$ & $10.2(4)$ & 0.0 & $12.3(2)$ & $6.1(5)$ & $7.1(4)$ \\
\hline 3 & Vegetables & 45.1 & $19.8(0.6)$ & 1.5 & 1.8 & 1.4 & 0.6 & 3.5 & 2.9 & $15.5(3)$ & $21.8(3)$ & $4.4(5)$ & 3.9 & 1.5 \\
\hline 4 & $\begin{array}{l}\text { Sugar sweetened } \\
\text { beverages }\end{array}$ & 42.6 & $50.1(1.9)^{1}$ & $4.5(4)$ & $5.9(3)$ & 1.5 & 1.8 & $18.9(2)$ & $10.5(2)$ & $15.8(2)$ & $36.2(1)$ & $9.6(4)$ & $10.1(2)$ & $9.4(3)$ \\
\hline 5 & $\begin{array}{l}\text { beverages } \\
\text { Cow's milk }\end{array}$ & 31.5 & $16.1(0.8)^{2}$ & $5.4(2)$ & $2.7(5)$ & $8.6(3)$ & $12.5(2)$ & $6.6(4)$ & $27.9(1)$ & $20.9(1)$ & $7.5(4)$ & $31.9(1)$ & 1.5 & $6.3(5)$ \\
\hline 6 & Table sugar & 30.0 & $3.0(0.1)$ & 1.2 & 1.8 & 0.0 & 0.0 & 0.0 & 0.2 & 0.0 & 0.0 & 1.1 & 0.2 & 0.0 \\
\hline 7 & Bread & 29.3 & $15.9(0.6)$ & $5.3(3)$ & $6.0(2)$ & 5.4 & 2.9 & $7.2(5)$ & 3.5 & 2.1 & 0.0 & 2.7 & $9.2(4)$ & 4.0 \\
\hline 8 & Eggs & 26.5 & $9.8(0.4)$ & 1.4 & 0.1 & 4.0 & 4.3 & 1.4 & $6.1(4)$ & 6.4 & 0.0 & 1.2 & 3.1 & 3.8 \\
\hline 9 & Noodles & 24.8 & $8.8(0.4)$ & $3.9(5)$ & $3.4(4)$ & 2.8 & $6.2(4)$ & 6.1 & 2.0 & 0.4 & 0.4 & 0.7 & 2.9 & 2.8 \\
\hline 10 & Pork & 20.8 & $11.2(0.7)$ & 3.5 & 0.2 & $6.0(5)$ & $12.7(1)$ & $8.1(3)$ & 3.9 & 5.3 & 0.6 & 1.0 & 2.9 & $10.5(2)$ \\
\hline 11 & Sausages/luncheon & 17.3 & $11.7(0.8)$ & 3.1 & 0.5 & 5.3 & $10.3(3)$ & 2.2 & 2.5 & 2.2 & 0.0 & 1.4 & $9.3(3)$ & 6.1 \\
\hline 12 & $\begin{array}{l}\text { meats } \\
\text { Cookies }\end{array}$ & 17.2 & $5.4(0.4)$ & 2.6 & 2.4 & 1.1 & $4.2(5)$ & 1.2 & 1.1 & 1.0 & 0.0 & 1.3 & 1.7 & 0.9 \\
\hline 13 & Chicken & 17.1 & $9.6(0.6)$ & 1.5 & 0.0 & $6.1(4)$ & 3.4 & 1.6 & 3.5 & $7.9(5)$ & $1.3(5)$ & 1.6 & 2.3 & 3.6 \\
\hline 14 & Fruits & 16.7 & $14.3(1.3)$ & 1.3 & 1.7 & 0.5 & 0.4 & 1.4 & 1.1 & 1.3 & $23.6(2)$ & 1.3 & 1.8 & 0.7 \\
\hline 15 & Cakes & 13.6 & $6.6(0.6)$ & 2.5 & 2.4 & 1.2 & 3.7 & 2.2 & 0.9 & $\begin{array}{l}2.8 \\
2.8\end{array}$ & 0.0 & 2.2 & 2.7 & 1.2 \\
\hline 16 & Crackers & 12.6 & $3.7(0.2)$ & 1.8 & 1.5 & 1.1 & 3.5 & 1.5 & 1.0 & 0.0 & 0.0 & 0.8 & 1.2 & 0.8 \\
\hline 17 & Candy & 9.7 & $1.9(0.2)$ & 0.9 & 0.8 & 0.5 & 1.5 & 0.3 & 0.5 & 0.3 & 0.0 & 1.4 & 0.6 & 0.9 \\
\hline 18 & Beans, nuts \& peas & 8.5 & $2.3(0.4)$ & 0.9 & 0.6 & 1.5 & 1.2 & 1.8 & 0.6 & 0.1 & 0.5 & 0.9 & 1.5 & 1.1 \\
\hline 19 & Savory snacks & 8.2 & $2.0(0.5)$ & 0.8 & 0.7 & 0.3 & 1.6 & 0.3 & 0.2 & 0.2 & 0.0 & 0.6 & 0.8 & 0.6 \\
\hline & Sweet biscuits & 6.5 & $2.1(0.2)$ & 1.0 & 0.9 & 0.4 & 1.7 & 0.5 & 0.3 & 0.9 & 0.0 & 0.6 & 0.7 & 0.1 \\
\hline \multicolumn{2}{|c|}{ Total contribution of top 20 foods } & & & 87.1 & 89.1 & 90.2 & 78.6 & 88.9 & 82.9 & 93.4 & 91.9 & 88.0 & 84.2 & 87.2 \\
\hline
\end{tabular}

${ }^{1}$ includes $88.6 \%$ liquid beverages and $11.4 \%$ beverage powder. ${ }^{2}$ includes $36.6 \%$ liquid milk and $63.4 \%$ milk powder. Grey shadow and bold words highlight the top-five food sources of nutrients. 


\section{Discussion}

Our analyses focused on nutrient intakes from food and beverages only to provide important information to inform compliance with food-based dietary guidance or to develop interventions to better meet nutrient needs. The data provide an overview of dietary patterns among Filipino infants, toddlers and young children and demonstrate that the diet of this population group is far from optimal. To our knowledge, this is the first study to provide current and comprehensive estimates of usual intakes of nutrients and food sources of key nutrients in children under 5 years old in the Philippines. In addition, the finding of this study could also provide insights to plan similar studies in other countries in Southeast Asia, where the diets of children are also largely based on rice and malnutrition remains a pressing issue.

\subsection{Inadequate Nutrient Intakes}

We examined the prevalence of inadequate nutrient intakes relative to the EAR when available and percentile distribution and mean intakes were presented for the rest of the nutrients. The results demonstrated that a high prevalence of inadequate intakes were found for all nutrients evaluated except for selenium. The prevalence of inadequate intakes ranged from $60 \%$ to $90 \%$ for iron, calcium, vitamin C, folate and zinc and from 30\% to $50 \%$ for other nutrients. In addition, no age group met the AIs for vitamin E, vitamin D or potassium, implying a high risk of inadequacy for these nutrients as well. Our results are in line with previous studies and surveys, in which large shortfalls in nutrient intakes were reported in the Philippines, although there were differences in the methodology of nutrient intake assessment $[7,28,29]$.

The period from birth to age five is a critical window for optimal growth and development [3]. Our results suggest that there are large shortfalls in the diet of this population and this could be one of the factors contributing to the high prevalence of underweight, stunting and nutrition deficiencies $[11,30]$.

\subsection{Low Food Variety and Diets Lack Nutrient-Dense Foods}

As an infant grows and develops, it experiences physiological shifts in nutrient and energy requirements that can no longer be supported by breast milk alone. Therefore, complementary foods with relative high energy and nutrient density and of good variety must be provided [31]. In this population, we found that the majority of the children consumed few foods, many of which were low in nutrients, such as rice, cookies and sugar.

Breast milk was consumed by $60 \%$ of infants (6-11.9 months) and $37 \%$ of toddlers (12-23.9 months). This is suboptimal based on breastfeeding guidelines of the World Health Organization [32] but these rates are higher than for infants and toddlers in studies conducted in China and the United States $[27,33]$. Breast milk was indeed a main source of all nutrients in infants 6-11.9 months and toddlers 12-23.9 months in this study. Other milk sources including infant formula and toddler/preschooler formula in infants and toddlers and cow's milk in young children also played important roles in providing nutrients. Another important finding is the role of fish in the diet. Fish was the second most consumed food among toddlers 24-35.9 months and young children 36-59.9 months, and was an important food source for a number of key nutrients including protein, calcium, iron, zinc, vitamin $\mathrm{A}$, and riboflavin.

However, the fact that refined rice was the first source of energy and the top source of many key nutrients in all age groups indicates that besides milk and fish, other nutrient-rich foods are missing from the diet. Indeed, we found that overall vegetables, fruit, and meats were only consumed by a small proportion of children and contributed little to nutrient intake. For example, fruits were only consumed by $5 \%$ of the infants and 14 to $17 \%$ of toddlers and young children. Vegetable consumption was as low as $11 \%$ among infants, in other words, only 1 in 10 of the infants consumed vegetables. Although older children consumed more vegetables, the quantity was less than 20 grams per capita per day in young children aged 36-59.9 months. In addition, other nutrient-rich foods such as meats, eggs 
and infant cereals were not commonly consumed either. About 10 to $25 \%$ of the children consumed eggs and meats. Infant cereal was consumed by $14 \%$ of the infants. Infant cereal is fortified with iron and other nutrients, a complementary food recommended by nutrition and medical organizations in many countries $[3,34]$. In fact, our data show that infant cereal was among the top food sources of almost all nutrients, although it was only consumed by a small proportion of the children.

Another unique observation is that sugar-sweetened beverages were among the top sources of nutrients in the diets of these children. In Western countries, sugar-sweetened beverages are discouraged by health professionals due to high sugar content [3]. In the Philippines, sugar-sweetened beverages are affordable and are among commonly consumed foods. Therefore, sugar-sweetened beverages are often used as carriers for fortification [35-37]. This makes sugar-sweetened beverages sources of micronutrients. We could not evaluate added sugar intake in this study because the data are not currently available in the Philippine FCT database.

\subsection{Strengths and Limitations}

This study provides one of the largest, population-based sources of data on usual energy and nutrient intakes from children aged 6 months up to 59.9 months. Data are presented by short age intervals to capture shifts in dietary patterns during this period of rapid growth and development. This study has several limitations. First, we examined intake on a given day, therefore, we may have underestimated the consumption of foods that are not consumed on a daily basis. Second, this study relied on mother or caregiver reports of child intake. The participants may have overor under-estimated their child's consumption during the recall [38]. However, because of the large sample size with corresponding survey weights applied in all the datasets, these limitations could be overshadowed and could represent valuable national information. Third, because the purpose of this study was to estimate nutrient intakes from food and beverages only, the use of dietary supplements were not included. This could lead to underestimation of total daily intakes of micronutrients in these children. A further study that assesses nutrient intakes from foods, beverages and dietary supplements is warranted. Lastly, about half of the data in the food composition database was built by adopting data from the National Nutrient Database of United States Department of Agriculture and from some other nearby countries. Although efforts were made to select the foods that could match the equivalent foods in the Philippines, food composition of foods from other countries may not reflect the foods in the Philippines. This could also induce over- or underestimation of nutrient intakes in this population.

\section{Conclusions}

This study provided important insights into the dietary patterns among Filipino children aged 6-59.9 months. The results showed that the intakes of total fat as a percentage of energy and most of micronutrients were highly inadequate. This can be explained largely by the low variety and low nutritional quality of foods consumed in this population. The diets of these children were made of few foods, namely the majority of energy and nutrients come from refined rice and other low nutrient-dense foods (cookies and sugar), while vegetables, fruits, meats and eggs made little contribution. The findings from this study should provide direction to healthcare professionals developing food-based recommendations and may contribute to intervention strategies to tackle the shortfalls in the diet of this population. Further studies to understand factors influencing the dietary intake of this population, such as socioeconomic status of families, food consumption habits and food access in different resigns are underway. In addition, the findings and insights obtained from this study could be shared with the nutrition researchers and healthcare professionals in other countries in Southeast Asia, where malnutrition of children is prevalent.

Author Contributions: L.D. contributed to data analysis, was responsible for data interpretation and writing the manuscript, and had final responsibility for this manuscript. I.A.-A. contributed to data analysis, data interpretation and manuscript editing. M.V.C. contributed to the critical review and editing of the manuscript. 
M.B.T. and J.D. contributed to building of food composition tables and food groups and data analysis. A.C. contributed to data analysis and data interpretation. All authors read and approved the final manuscript.

Funding: This research was funded by Nestle Institute of Health Sciences, Lausanne, Switzerland.

Acknowledgments: We acknowledge the contribution of Virgilyn Anne Obligar, Royce Ann Octavio, Kristine Biona and Regina Rodriguez in building food composition tables and food group. We thank Mark Lester Cayadong and Glen Melvin Gironella for their work in data management and programming. We would like to thank the field workers who assisted with the data collection and the mothers and children who participated in the study.

Conflicts of Interest: The authors declare no conflict of interest. L.D. is an employee of Nestec Ltd. (Nestlé Institute of Health Sciences), Lausanne, Switzerland. The opinions expressed in the article are those of the authors alone and do not necessary reflect the views of recommendations of their affiliations.

\section{References}

1. Dewey, K.G.; Begum, K. Long-term consequences of stunting in early life. Matern. Child. Nutr. 2011, 7, 5-18. [CrossRef] [PubMed]

2. Butte, N.; Cobb, K.; Dwyer, J.; Graney, L.; Heird, W.; Rickard, K.; American Dietetic Association; Gerber Products Company. The start healthy feeding guidelines for infants and toddlers. J. Am. Diet. Assoc. 2004, 104, 442-454. [CrossRef] [PubMed]

3. American Academy of Pediatrics. Pediatric Nutrition; American Academy of Pediatrics: Itasca, IL, USA, 2014.

4. The World Bank. Philippines Economic Update: Investing in the Future; The World Bank: Washington, DC, USA, 2018; pp. 2-5.

5. Food and Nutrition Research Institute. Facts and Figures 2013, 8th National Nutrition Survey Anthropometric Survey; Food and Nutrition Research Institute, Department of Science and Technology: Metro Manila, Philippines, 2015; pp. 13-28.

6. Food and Nutrition Research Institute. Facts and Figures 2013, 8th National Nutrition Survey Biochemical Survey; Food and Nutrition Research Institute, Department of Science and Technology: Metro Manila, Philippines, 2015; pp. 23-51.

7. Perlas, L.A.; Gibson, R.S.; Adair, L.S. Macronutrient and selected vitamin intakes from complementary foods of infants and toddlers from Cebu, Philippines. Int. J. Food Sci. Nutr. 2004, 55, 1-15. [CrossRef] [PubMed]

8. Rohner, F.; Woodruff, B.A.; Aaron, G.J.; Yakes, E.A.; Lebanan, M.A.; Rayco-Solon, P.; Saniel, O.P. Infant and young child feeding practices in urban Philippines and their associations with stunting, anemia, and deficiencies of iron and vitamin A. Food Nutr. Bull. 2013, 34, S17-S34. [CrossRef] [PubMed]

9. Wright, M.J.; Bentley, M.E.; Mendez, M.A.; Adair, L.S. The interactive association of dietary diversity scores and breast-feeding status with weight and length in Filipino infants aged 6-24 months. Public Health Nutr. 2015, 18, 1762-1773. [CrossRef] [PubMed]

10. Kennedy, G.L.; Pedro, M.R.; Seghieri, C.; Nantel, G.; Brouwer, I. Dietary diversity score is a useful indicator of micronutrient intake in non-breast-feeding Filipino children. J. Nutr. 2007, 137, 472-477. [CrossRef] [PubMed]

11. Tengco, L.W.; Rayco-Solon, P.; Solon, J.A.; Sarol, J.N., Jr.; Solon, F.S. Determinants of anemia among preschool children in the Philippines. J. Am. Coll. Nutr. 2008, 27, 229-243. [CrossRef] [PubMed]

12. Chaparro, C.; Oot, L.; Sethuraman, K. Overview of the Nutrition Situation in Seven Countries in Southeast Asia; Food and Nutrition Technical Assistance III P: Washington, DC, USA, 2014; pp. 4-24.

13. Food and Nutrition Research Institute. Facts and Figures 2015, 8th National Nutrition Survey Overview; Food and Nutrition Research Institute, Department of Science and Technology: Metro Manila, Philippines, 2015; p. 5.

14. World Health Organization Department of Nutrition for Health and Development. Who Child Growth Standards; World Health Organization: Geneva, Switzerland, 2006.

15. Food and Nutrition Research Institute, Department of Science and Technology. The Philippine Food Composition Tables 1997; Department of Science and Technology, Food and Nutrition Research Institute: Manila, Philippines, 1997.

16. International Network of Food Data Systems (INFOODS). Standards and Guidelines. Available online: http:/ / www.fao.org/infoods/infoods/standards-guidelines/en/ (accessed on 26 May 2017).

17. Lopez-Olmedo, N.; Carriquiry, A.L.; Rodriguez-Ramirez, S.; Ramirez-Silva, I.; Espinosa-Montero, J.; Hernandez-Barrera, L.; Campirano, F.; Martinez-Tapia, B.; Rivera, J.A. Usual intake of added sugars and saturated fats is high while dietary fiber is low in the Mexican population. J. Nutr. 2016, 146, 1856S-1865S. [CrossRef] [PubMed] 
18. Institute of Medicine. Dietary Reference Intakes for Energy, Carbohydrate, Fiber, Fat, Fatty Acids, Cholesteroal, Protein, and Amino Acids; The National Academies Press: Washington, DC, USA, 2005.

19. Devaney, B.; Ziegler, P.; Pac, S.; Karwe, V.; Barr, S.I. Nutrient intakes of infants and toddlers. J. Am. Diet. Assoc. 2004, 104, 14-21. [CrossRef] [PubMed]

20. Butte, N.F.; Fox, M.K.; Briefel, R.R.; Siega-Riz, A.M.; Dwyer, J.T.; Deming, D.M.; Reidy, K.C. Nutrient intakes of us infants, toddlers, and preschoolers meet or exceed dietary reference intakes. J. Am. Diet. Assoc. 2010, 110, S27-S37. [CrossRef] [PubMed]

21. Food and Nutrition Research Institute Department of Science and Techology. Annual Report 2017; Food and Nutrition Research Institute: Manila, Philippines, 2018.

22. Siega-Riz, A.M.; Deming, D.M.; Reidy, K.C.; Fox, M.K.; Condon, E.; Briefel, R.R. Food consumption patterns of infants and toddlers: Where are we now? J. Am. Diet. Assoc. 2010, 110, S38-S51. [CrossRef] [PubMed]

23. Fox, M.K.; Condon, E.; Briefel, R.R.; Reidy, K.C.; Deming, D.M. Food consumption patterns of young preschoolers: Are they starting off on the right path? J. Am. Diet. Assoc. 2010, 110, S52-S59. [CrossRef] [PubMed]

24. Institute, F.A.N.R. Philippine Dietary Reference Intakes 2015; Food and Nutrition Research Institute, Department of Science of Technology: Manila, Philippines, 2015.

25. Carriquiry, A.L. Assessing the prevalence of nutrient inadequacy. Public Health Nutr. 1999, 2, $23-33$. [CrossRef] [PubMed]

26. National Research Council. Nutrient Adequacy: Assessment Using Food Consumption Surveys; National Academy Press: Washington DC, USA, 1986.

27. Yu, P.; Denney, L.; Zheng, Y.; Vinyes-Pares, G.; Reidy, K.C.; Eldridge, A.L.; Wang, P.; Zhang, Y. Food groups consumed by infants and toddlers in urban areas of China. Food Nutr. Res. 2016, 60, 30289. [CrossRef] [PubMed]

28. Angeles-Agdeppa, I.; Gironella, G.; Constantino, M. Evaluation of calcium intakes of young children in the Philippines as a result of the 2008 national nutrition survey. Philipp. J. Sci. 2016, 145, 165-174.

29. Food and Nutrition Research Institute. National Nutrition Survey Facts and Figures; Food and Nutrition Research Institute Department of Science and Technology: Manila, Philippines, 2008.

30. Walker, S.P.; Wachs, T.D.; Gardner, J.M.; Lozoff, B.; Wasserman, G.A.; Pollitt, E.; Carter, J.A.; International Child Development Steering Group. Child development: Risk factors for adverse outcomes in developing countries. Lancet 2007, 369, 145-157. [CrossRef]

31. U.S. Department of Agriculture. Scientific Report of the 2015 Dietary Guidelines Advisory Committee: Advisory Report to the Secretary of Health and Human Services and the Secretary of Agriculture; U.S. Department of Agriculture, Agricultural Research Service: Washington, DC, USA, 2015.

32. WHO. Global Strategy for Infant and Young Child Feeding; World Health Organization: Geneva, Switzerland, 2003.

33. Roess, A.A.; Jacquier, E.F.; Catellier, D.J.; Carvalho, R.; Lutes, A.C.; Anater, A.S.; Dietz, W.H. Food consumption patterns of infants and toddlers: Findings from the feeding infants and toddlers study (FITS) 2016. J. Nutr. 2018, 148, 1525S-1535S. [CrossRef] [PubMed]

34. Child Health Study Group. Infant feeding recommendation. Chin. J. Pediatr. 2009, 47, 504-507.

35. Solon, F.S. Food fortification in the Philippines: Policies, programmes, issues, and prospects. Food Nutr. Bull. 2000, 21, 515-520. [CrossRef]

36. Angeles-Agdeppa, I.; Magsadia, C.R.; Aaron, G.J.; Lloyd, B.B.; Hilmers, D.C.; Bhutta, Z.A. A micronutrient fortified beverage given at different dosing frequencies had limited impact on anemia and micronutrient status in Filipino schoolchildren. Nutrients 2017, 9. [CrossRef] [PubMed]

37. Angeles-Agdeppa, I.; Magsadia, C.R.; Capanzana, M.V. Fortified juice drink improved iron and zinc status of schoolchildren. Asia Pac. J. Clin. Nutr. 2011, 20, 535-543. [PubMed]

38. Burrows, T.L.; Martin, R.J.; Collins, C.E. A systematic review of the validity of dietary assessment methods in children when compared with the method of doubly labeled water. J. Am. Diet. Assoc. 2010, 110, 1501-1510. [CrossRef] [PubMed]

(C) 2018 by the authors. Licensee MDPI, Basel, Switzerland. This article is an open access article distributed under the terms and conditions of the Creative Commons Attribution (CC BY) license (http://creativecommons.org/licenses/by/4.0/). 